\title{
Internalization routes of cell-penetrating melanoma antigen peptides into human dendritic cells
}

\author{
Timo Buhl ${ }^{1}$, Andrea Braun ${ }^{1}$, Susann Forkel ${ }^{1}$, Wiebke Möbius ${ }^{2,3}$, Lars van Werven ${ }^{4}$, Olaf Jahn ${ }^{4}$, Nasrollah \\ Rezaei-Ghaleh ${ }^{5,6}$, Markus Zweckstetter ${ }^{3,5,6}$, Martin Mempel ${ }^{1}$, Michael P. Schön ${ }^{1}$ and Holger A. Haenssle ${ }^{1}$ \\ ${ }^{1}$ Clinic of Dermatology, Venereology and Allergology, University Medical Center Göttingen, Göttingen, Germany; ${ }^{2}$ Department of Neurogenetics, \\ Max Planck Institute of Experimental Medicine, Göttingen, Germany; ${ }^{3}$ Center for Nanoscale Microscopy and Molecular Physiology of the Brain \\ (CNMPB), University Medical Center Göttingen, Göttingen, Germany; ${ }^{4}$ Proteomics Group, Max Planck Institute of Experimental Medicine, \\ Göttingen, Germany; ${ }^{5}$ Max Planck Institute for Biophysical Chemistry, Göttingen, Germany; ${ }^{6}$ German Center for Neurodegenerative Diseases \\ (DZNE), Göttingen, Germany \\ Correspondence: Timo Buhl, MD, Clinic of Dermatology, Venereology and Allergology, Georg August University Göttingen, Robert-Koch-Str. 40, \\ D-37075 Göttingen, Germany, Tel.: +49-551-396410, Fax: +49-551-3922047, e-mail: timo.buhl@med.uni-goettingen.de
}

Abstract: Optimized delivery of antigens combined with sustainable maturation of dendritic cells (DCs) is crucial for generation of effective antitumoral immune responses. Multiple approaches for ex vivo antigen loading and improvement in immunogenicity have been described. We have recently established a single-step protocol consisting of a fusion peptide (a sequence of the melanoma antigen Melan-A and a cationic cellpenetrating HIV TAT domain) bound in complexes with a tolllike receptor agonist. As the exact cellular uptake mechanisms of TAT-coupled antigens have been a matter of considerable debate and significantly depend on cell type, cargo and concentrations, we evaluated internalization routes into human immature DCs in comparison with non-phagocytic cell lines. We found that MelanA-TAT fusion peptide uptake by DCs is mainly energy dependent, superior compared with polylysine-coupled Melan-A and significantly higher in DCs as compared with Jurkat cells or HUVECs. Furthermore, we could track the uptake of the fusion peptide exclusively through early endosomes to lysosome compartments after 90 min by fluorescence microscopy and immunoelectron microscopy. Specific endocytosis inhibitors revealed major internalization of the fusion peptide by DCs via clathrin-mediated endocytosis, whereas uptake by non-phagocytic HUVECs differed significantly, involving macropinocytosis as well as clathrin-mediated endocytosis. As our understanding of the processes involved in internalization of TAT-coupled cargos by human DCs broadens, and DC activation becomes available by single-step procedures as described, further development of simultaneous DC maturation and intra-cellular peptide targeting is warranted.

Abbreviations: CPP, cell-penetrating peptide; $\mathrm{CPZ}$, chlorpromazine DC, dendritic cell; EEA, early endosomal antigen; EIPA, 5-(N-ethyl-Nisopropyl)amiloride; HLA, human leucocyte antigen; HUVEC, human umbilical vein endothelial cell; MbCD, methyl- $\beta$-cyclodextrin; MHC, major histocompatibility complex; OVA, ovalbumin; PBMC, peripheral blood mononuclear cell; Poly(i/c), polyinosinic/polycytidylic acid Th1, T helper cell type 1 .

Key words: cell-penetrating peptides - cellular immunotherapy dendritic cells - Melan-A/Mart-1 - TAT

Accepted for publication 11 November 2013

\section{Introduction}

Dendritic cells (DCs) are major players in mounting effective immune responses to cancer antigens. This phenomenon can be enhanced by DC-based immunotherapy $(1,2)$. Multiple protocols for delivery of antigens to DCs have been established (3). Exogenous HLA-matched peptides with high affinity may replace antigens from preformed major histocompatibility complex (MHC)-peptide complexes on the cell surface without cellular uptake (4). Alternatively, uptake of exogenous peptides and proteins by DCs with subsequent processing, loading and crosspresentation on MHC class I and II molecules may occur $(5,6)$. With regard to the high turnover of MHC-peptide complexes residing on the cell surface, only a small fraction of the initial MHC-peptide complexes (if any) will be present on DCs, which physiologically reached the draining lymph node after $24-72 \mathrm{~h}$ $(7,8)$. Therefore, intra-cellular uptake of defined antigens, prolonged storage in intra-cellular reservoirs, and permanently ongoing processing and cross-presentation on MHC molecules will be crucial for optimal antigen presentation on encounter of the DCs with T cells in the lymph node.
Cationic cell-penetrating peptides (CPPs) are a promising vehicle for the delivery of proteins and other molecules into cells and even across the blood-brain barrier, because CPPs possess the ability to cross plasma membranes, reach the cytoplasm and form intra-cellular reservoirs $(9,10)$. Therefore, CPPs have become intensively studied vectors in many research areas, including drug delivery, signal transduction, cancer, angiogenesis, apoptosis, bone development, cardio protection, cell cycle, neurobiology and many others (11). Besides their basic cell penetration capacities, CPPs have also significant immunological effects, because they can act as antimicrobial agents, leucocyte chemoattractants and inducers of T-cell receptors in tumor microenvironments (12). Recently, we have successfully established a fusion peptide consisting of a cationic HIV-1 TAT domain (one of the most widely used CPPs) and a MHC I binding melanoma antigen domain (Melan-A/ Mart-1) for ex vivo DC antigen loading (13).

Despite extensive studies following the discovery of the first CPPs, their exact cellular uptake mechanisms remain controversial (14-17). Cell-penetrating peptide side chains with special chemical properties have been studied for interaction, yet resulted in 
conflicting findings $(18,19)$. Some studies have indicated that CPPs can trigger their own uptake by stimulating endocytosis (20-22). Others suggested a modulation of uptake mechanisms by the type of the target cell, the cargo coupled to the CPP itself, or the amount of CPP-coupled molecules in contact with the target cell (23-25). Although the induction of an improved DC immunogenicity by CPP-coupled antigens has been evaluated by us and others (26-29), no studies have investigated the exact uptake mechanisms of CPPs in highly phagocytic human cells such as immature DCs so far.

\section{Materials and methods}

\section{PBMC preparation and DC generation}

Leukaphereses of healthy donors were obtained by a Cobe Spectra Auto PBSC device (Gambro BCT Inc., Lakewood, CA, USA) after the approval by local ethics committees and written informed consent according to institutional guidelines. Preparation of peripheral blood mononuclear cells (PBMCs) was performed by density gradient centrifugation; aliquots of isolated PBMCs were used for immediate cell culture or for cryopreservation, as described earlier (30). Immature DCs were generated from a plastic-adherent cell fraction of PBMCs. Adherent cells were grown in RPMI 1640 supplemented with $10 \%$ FCS, $100 \mathrm{IU} / \mathrm{ml}$ penicillin, $100 \mu \mathrm{g} / \mathrm{ml}$ streptomycin (PAA, Pasching, Austria), $800 \mathrm{IU} / \mathrm{ml}$ GM-CSF and $1000 \mathrm{IU} / \mathrm{ml}$ IL-4 (Immunotools, Friesoythe, Germany) and cultured for six days to differentiate into immature DCs (31). At this point, analyses for surface markers CD3, CD14, CD83, CD86 and HLA-DR were performed in all experiments for quality control of immature DCs.

\section{Peptide synthesis}

The Melan-A-TAT fusion peptide [CELAGIGILTVRKKRRQRRR, Melan-A (26-35) sequence underlined] was synthesized using standard fluorenylmethoxycarbonyl (Fmoc) chemistry. The N-terminal Cys residue was added to enable coupling of Alexa Fluor 488 (Alexa488) $C_{5}$ maleimide (Invitrogen, Carlsbad, CA, USA). Prior to coupling, Melan-A-TAT was purified by standard reversed-phase HPLC, and its identity was confirmed by mass spectrometry. The Alexa Fluor 488-tagged fusion peptide (Alexa488-Melan-A-TAT) was generated by mixing equal volumes of the peptide solution (2.1 mM in $50 \mathrm{~mm}$ Tris- $\mathrm{HCl} \mathrm{pH} \mathrm{7.4)} \mathrm{and}$ of the Alexa Fluor $488 \mathrm{C}_{5}$ maleimide solution $(7.5 \mathrm{~mm}$ in anhydrous DMSO). The mixture was kept under light exclusion and incubated for $2 \mathrm{~h}$ at room temperature on a shaker and, thereafter, was diluted $1: 5$ with $0.1 \%$ trifluoroacetic acid (TFA)/10\% acetonitrile and separated by reversed-phase HPLC on a C4 column (linear gradient of $0.4 \%$ acetonitrile/min from $0.1 \%$ TFA $/ 15 \%$ acetonitrile to $0.1 \%$ TFA $/ 40 \%$ acetonitrile). Purified Alexa488-MelanA-TAT was characterized by mass spectrometry using both MALDI and electrospray ionization as a partial loss of the Alexa488 group was observed under MALDI conditions. Alexa488Melan-A-polyLys (CELAGIGILTVKKKKKQKKK) was designed as control peptide.

\section{Dynamic light scattering (DLS)}

Dynamic light scattering experiments were performed at $20{ }^{\circ} \mathrm{C}$ on a DynaPro Titan (Wyatt Technology Corp., Santa Barbara, CA, USA) instrument with a laser of $827.08 \mathrm{~nm}$ and a scattering angle of 90 degrees. The peptide solution was prepared at a concentration of $200 \mu \mathrm{m}$ in $10 \mathrm{~mm}$ acetic acid and stored at $-20^{\circ} \mathrm{C}$ until further use. After thawing, the peptide solution was centrifuged at
$5000 \mathrm{~g}$ for $10 \mathrm{~min}$ at $4{ }^{\circ} \mathrm{C}$, and the supernatant was taken for immediate triplicate DLS measurements. Each DLS measurement consisted of twelve 20-s long acquisitions. The size distribution was determined by a constrained regularization method.

\section{Flow cytometry}

Antigen expression was determined using antibodies against the following antigens: CD3, CD14, CD83, CD86, HLA-DR or the respective isotype-matched controls (all Invitrogen except CD83Ab from Beckman Coulter, Krefeld, Germany). Cells were washed and immediately analysed on a FACS Canto II and DIVA software (BD Biosciences, San Jose, CA, USA). Dead cells and debris were gated out on the basis of their light scatter properties. Integrity of the plasma membrane and thereby cell viability was assessed by propidium iodide staining (Sigma-Aldrich, St. Louis, MO, USA).

\section{Inhibition assay of TAT peptide uptake by decreased temperature and ATP depletion}

For depletion of the cellular ATP pool, immature DCs were preincubated for $60 \mathrm{~min}$ with $10 \mathrm{~mm}$ sodium azide (Merck-Schuchardt, Hohenbrunn, Germany) and $6 \mathrm{~mm}$ 2-deoxy-D-glucose (Sigma-Aldrich) and washed afterwards in RPMI. Alexa488Melan-A-TAT was added to each well (containing $3 \times 10^{5} \mathrm{DCs} /$ $1 \mathrm{ml} \mathrm{RPMI}$ ) for $30 \mathrm{~min}$ either at 4 or $37^{\circ} \mathrm{C}$. After two washings with trypsin (PAA), cells were immediately analysed by flow cytometry. For quality control of generated immature DCs, flow cytometry analyses of CD14, CD83, CD86 and HLA-DR were also performed for every experiment.

\section{Uptake assay of peptides and ovalbumin by DCs, HUVECs} and Jurkat cells

Human umbilical vein endothelial cells (HUVECs; Lonza, Basel, Switzerland) were cultured in endothelial cell growth medium (Lonza) to confluence. Twenty-four hours before an experiment, growth medium was replaced by endothelial basal medium (Lonza) supplemented with 10\% FCS (PAA). Disaggregation was achieved by the treatment with Accutase (PAA). Immature DCs, HUVECs and Jurkat cells were harvested, washed and transferred to $15-\mathrm{ml}$ Falcon tubes (Greiner Bio-One, Frickenhausen, Germany) at a density of $3 \times 10^{5}$ cells in $1 \mathrm{ml}$ RPMI. For the different experiments, $3 \mu \mathrm{g} / \mathrm{ml}$ Alexa488-Melan-A-TAT, $3 \mu \mathrm{g} / \mathrm{ml}$ Alexa488-MelanA-polyLys or $1 \mu \mathrm{g} / \mathrm{ml}$ OVA-FITC (Biosearch Technologies, Novato, CA, USA) were added as antigen, and cells were cultured at $37^{\circ} \mathrm{C}$ for $60 \mathrm{~min}$. After two washings with trypsin $(1 \mathrm{mg} / \mathrm{ml})$, cells were immediately analysed by flow cytometry.

\section{Immunoelectron microscopy}

After the uptake of Alexa488 labelled peptide for 30 or $90 \mathrm{~min}$, immature DCs were fixed in $4 \%$ formaldehyde and $0.2 \%$ glutaraldehyde in $0.1 \mathrm{~m}$ phosphate buffer. Then, the cells were washed and scraped off the dish in $0.1 \mathrm{~m}$ phosphate buffer containing $1 \%$ gelatin, spun down and resuspended in $10 \%$ gelatin in $0.1 \mathrm{~m}$ phosphate buffer at $37^{\circ} \mathrm{C}$. After spinning down, the pellets in gelatin were cooled on ice, removed from the tubes and cut in small blocks. These blocks were infiltrated in $2.3 \mathrm{M}$ sucrose in $0.1 \mathrm{M}$ phosphate buffer, mounted onto aluminium pins for ultramicrotomy and frozen in liquid nitrogen. Ultrathin cryosections were prepared with a Leica UC6 cryo-ultramicrotome (Leica, Vienna, Austria) using a cryo-diamond knife from Diatome (Biel, Switzerland). For double immunolabelling, sections were incubated with antibodies specific for LAMP1 (raised in rabbit; Biolegend, San 
Diego, CA, USA), which was detected with protein A-gold (15 nm, from CMC, Utrecht, the Netherlands) followed by incubation with Anti-Alexa488 (raised in rabbit; Invitrogen), which was detected with protein A-gold (10 nm, from CMC). Sections from cells without peptide uptake served as negative control for the Alexa488 labelling. Labelled sections were analysed with a LEO EM912 Omega electron microscope (Zeiss, Oberkochen, Germany), and digital micrographs were obtained with an on-axis $2048 \times 2048$-CCD camera (TRS, Moorenweis, Germany).

\section{Immunofluorescence microscopy}

For analysis of subcellular compartments by immunofluorescence microscopy, Alexa488-peptides and Lysotracker (Invitrogen) were co-incubated with DCs at the same time at $37{ }^{\circ} \mathrm{C}$ for a 30 or 90 min time interval. After washing with trypsin, cells were fixed by $4 \%$ paraformaldehyde (Roth, Karlsruhe, Germany) and stained for EEA1 (Abcam, Cambridge, UK) or corresponding controls. Finally, cells were stained with DAPI (Sigma-Aldrich) and cytospins were performed. Cells were analysed by immunofluorescence microscopy using a Zeiss Imager M1 Axio (Carl Zeiss, Oberkochen, Germany).

Inhibition assay of antigen uptake by endocytosis inhibitors Cells were harvested and transferred to $15-\mathrm{ml}$ Falcon tubes at a density of $3 \times 10^{5}$ cells in $1 \mathrm{ml}$ RPMI. The phagocytosis inhibitors chlorpromazine (CPZ), 5-(N-ethyl-N-isopropyl)amiloride and methyl- $\beta$-cyclodextrin (MbCD) (all Sigma-Aldrich) were added for $30 \mathrm{~min}$ at $37^{\circ} \mathrm{C}$. Subsequently, medium was removed and fresh medium containing peptides or ovalbumin as well as the corresponding inhibitor were added (concentration of peptides identical as above). After incubation for 30 or $60 \mathrm{~min}$ at $37^{\circ} \mathrm{C}$, the cells were washed twice with medium and analysed by flow cytometry and fluorescence microscopy.

\section{Statistical analysis}

All figures show mean \pm standard error of observed values. The significance of the effects of the various experiments as compared with untreated controls was evaluated by the paired Student's $t$ test at the 95\% confidence interval. All analyses were performed using SAS 9.2 (SAS Institute Inc., Cary, NC, USA) software.

\section{Results}

\section{Peptide design and characteristics}

As in our previous studies on Melan-A-TAT fusion peptides, we used the peptide ELAGIGILTVRKKRRQRRR (13). Synthesis of an Alexa488-coupled acidic control fusion peptide (Melan-A-FLAG, ELAGIGILTVDYKDDDDK) failed due to its insolubility under coupling conditions. Therefore, we chose Melan-A-polyLys ELAGIGILTVKKKKKQKKK (replacing the six arginines of the TAT peptide by lysines only) as control peptide with similar biophysical properties compared with TAT. Lysine-rich peptides may also display minor CPP properties, but their uptake has been shown to be significantly lower compared with arginine-rich peptides such as TAT (19). Uncoupled Melan-A was not investigated here because this project focuses on the uptake mechanisms of Melan-A-TAT into DCs, and we have extensively studied the immunological benefit of CPP coupling of Melan-A peptides previously (13).

The size distribution of Alexa488-Melan-A-TAT, as revealed by DLS experiments, is presented in Fig. S1. Besides the peak at approximately $0.7 \mathrm{~nm}$ (possibly originating from the single Alexa488-Melan-A-TAT peptide), the peptide stock solution contained aggregates at approximately 35 and $100 \mathrm{~nm}$ hydrodynamic radii, which contributed roughly $7 \%, 8 \%$ and $85 \%$ to the total scattering intensity, respectively. Considering that the scattering intensity dramatically increases with the size of aggregates, it is reasonable to assume that a sizeable fraction of peptide mass (much more than 15\%) is represented by the two smaller categories of particle size.

Uptake of Melan-A-TAT peptide by human immature DCs is predominantly ATP and temperature dependent

Several studies suggested that direct penetration of the plasma membrane via energy-independent pathways plays a major role in TAT uptake. Hence, we studied the effects of ATP depletion and low temperature on Alexa488-Melan-A-TAT uptake by human immature DCs. The cells were cultured with Alexa488-Melan-ATAT for $30 \mathrm{~min}$, and we evaluated the amount of internalized peptide by flow cytometry (Fig. 1a,c). Uptake of the fusion peptide was reduced by more than $75 \%$ at $4{ }^{\circ} \mathrm{C}$ in comparison with an identical experimental set-up at $37^{\circ} \mathrm{C}$ (Fig. 1c). Pre-incubation of DCs with sodium azide and deoxyglucose for depletion of the cellular ATP pool resulted in a lesser, but still significant inhibition of fusion peptide uptake. As ATP depletion inhibited the uptake of Alexa488-Melan-A-TAT only partly, a second nonenergy-dependent uptake mechanism may be involved. ATP and temperature dependence of fusion peptide uptake by DCs is suggestive for a predominantly active, phagocytotic internalization pathway.
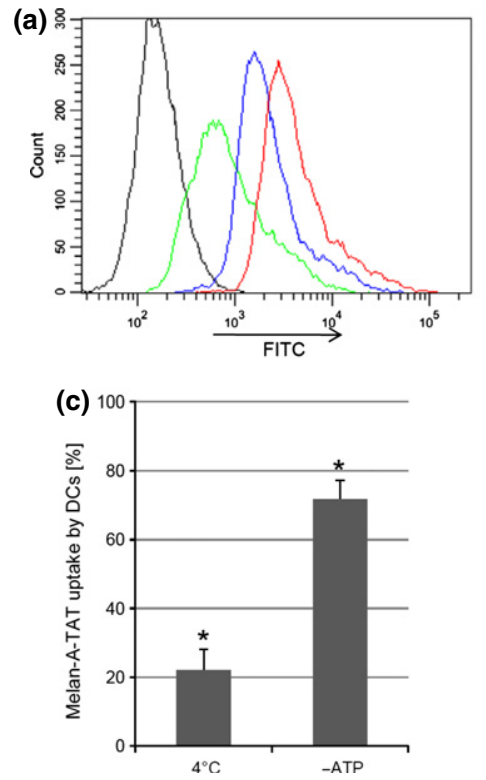

(b)
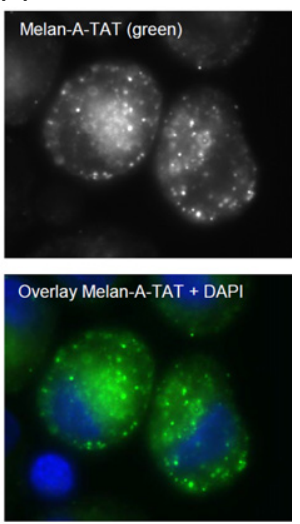

Figure 1. (a) Assessment of peptide internalization by flow cytometry. Dendritic cells (DCs) were incubated for 30 min with Alexa488-Melan-A-TAT at $37^{\circ} \mathrm{C}$ (red curve) or at $4{ }^{\circ} \mathrm{C}$ (green curve), or after depletion of the cellular ATP pool at $37^{\circ} \mathrm{C}$ (blue curve). The black curve represents DCs incubated in the absence of peptide. (b) Fluorescence microscopy image of Alexa488-Melan-A-TAT uptake by DCs after 90 min (upper image peptide only, lower image overlay peptide and DAPI staining). (c) Effect of $4{ }^{\circ} \mathrm{C}$ or depletion of the cellular ATP pool on Alexa488tagged Melan-A-TAT peptide internalization by DCs. Uptake is depicted as percentage of MFI compared with untreated controls' MFI incubated with Alexa488-Melan-A-TAT at $37^{\circ} \mathrm{C}$. Means \pm SD of three independent experiments are shown (cells obtained from three different donors). A significant reduction in uptake is marked with asterisks (paired $t$-test, $* P<0.05$ ). 
Uptake of Melan-A fusion peptides with TAT sequence is superior compared with a control polylysine sequence

To assess the effect of the TAT sequence within the fusion peptide, we designed a control fusion peptide consisting of Melan-A and a modified TAT sequence (Melan-A-polyLys). For comparison of the uptake of the Melan-A-TAT versus Melan-A-polyLys by DCs, cells were separately incubated with Alexa488-tagged fusion peptides for $30 \mathrm{~min}$. Thereafter, cells were harvested, washed twice with trypsin containing medium, and were analysed by flow cytometry. Uptake of Alexa488-Melan-A-TAT was significantly higher (approximately twofold) in all experiments compared with control peptide $(P<0.01)$ (Fig. 2, and detailed FACS plots in Fig. S2). Reduced but still observable uptake of the control peptide Alexa488-Melan-A-polyLys was expected due to its minor CPP properties compared with TAT. Interestingly, immature DCs of different healthy donors showed highly reproducible uptake results, despite the considerable interdonor variability of human DCs.

For comparison of cellular uptake mechanisms of DCs with other cell types, we also investigated the uptake of fusion peptides by HUVECs and Jurkat cells. Identical cell numbers and amounts of peptide, again, gave rise to a significantly superior uptake of Alexa488-Melan-A-TAT versus Melan-A-polyLys by these cells. As expected, the absolute level of antigen uptake for these nonphagocytotic cells was much lower than observed for immature DCs. In addition, we assessed uptake of FITC-coupled ovalbumin (OVA-FITC) by the different cells under identical conditions. Ovalbumin was readily internalized by immature DCs, whereas the rather non-phagocytic HUVECs and Jurkat cells completely failed to take up ovalbumin (Fig. 2).

Intra-cellular localization studies track Melan-A-TAT

peptides through early endosomes to lysosome compartments in DCs

Colocalization studies were performed by fluorescence microscopy at different time intervals to analyse the subcellular compartments involved in trafficking Alexa488-Melan-A-TAT. We could demonstrate that the fusion peptide is taken up into the lysosomal compartments of DCs $90 \mathrm{~min}$ after addition of the peptide to cultures (Fig. S3A). Visualization of Alexa488-Melan-A-TAT by

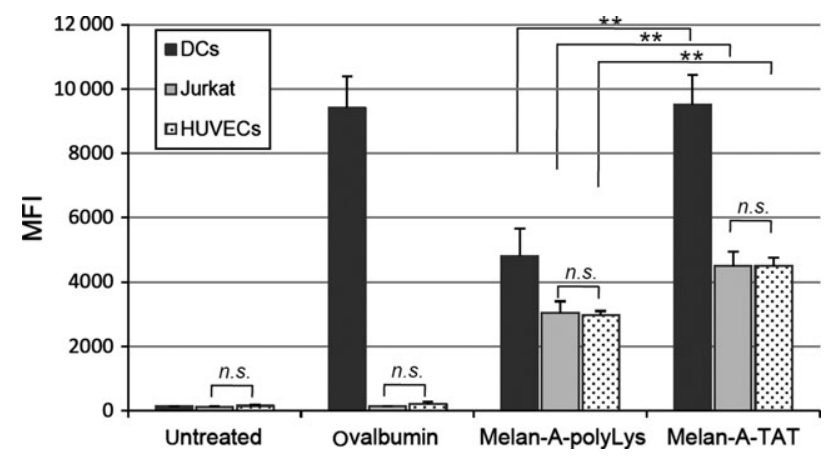

Figure 2. Quantification of ovalbumin-, Melan-A-polyLys- and Melan-A-TAT uptake by immature human dendritic cells (DCs), Jurkat cells and HUVECs. All cells were separately incubated for $60 \mathrm{~min}$ in the presence of OVA-FITC, Alexa488Melan-A-polyLys or Alexa488-Melan-A-TAT and analysed by flow cytometry [mean fluorescence intensity (MFI) depicted]. Experiments comparing Jurkat cells and HUVECs revealed no significant differences. Significance level $* * P<0.01$. All experiments $n=3$ (DCs from three different donors). fluorescence microscopy was limited during the first 15-20 min of cellular uptake due to low amounts of internalized peptide, hampering colocalization of the peptide with earlier subcellular compounds during peptide uptake (exemplified by EEA1 costaining after 30 resp. $90 \mathrm{~min}$ in Fig. S3B). For immunoelectron microscopy, the Alexa488-fluorophor was detected using anti-Alexa488 antibodies and protein A-gold to localize the peptide intra-cellular. Indeed, we could show that the Alexa488-Melan-A-TAT becomes internalized by the DC (Fig. 3a,b), then shuttled into early endosomes (Fig. 3c,d) and can be located in Lamp1-positive subcellular compartments (late endosomes/lysosomes) after $90 \mathrm{~min}$ (Fig. 3e, f). Remarkably, no peptide was found anywhere outside the endocytic compartments.

DCs facilitate uptake of Melan-A-TAT peptides by clathrinmediated endocytosis and use internalization routes other than those used by non-phagocytic cells

In order to identify specific uptake mechanisms of DCs, we investigated the uptake of Alexa488-Melan-A-TAT peptides and
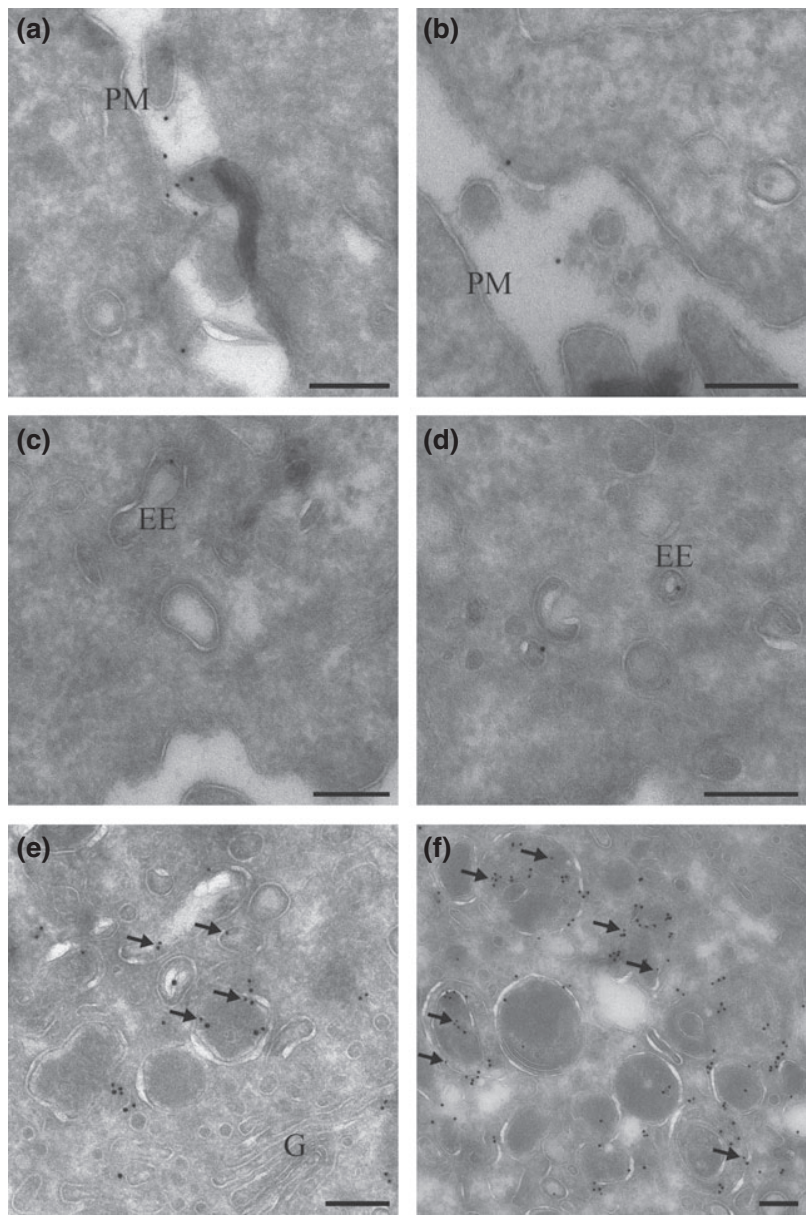

Figure 3. Immunoelectron microscopy reveals that uptake of Alexa488-coupled fusion peptides occurs in human immature dendritic cells by endocytosis. Scale in lower right corner of all pictures is always $250 \mathrm{~nm}$. In panel a, b, Alexa488coupled peptide is located at the cell surface (PM, plasma membrane), with some exosomes being present as well. Later as shown in $\mathrm{c}$, $\mathrm{d}$, the peptide can be visualized in early endosomes(EE). Panel e shows late endosomes/lysosomes containing the peptide by double-labelling of Lamp1 $(15 \mathrm{~nm})$ and fusion peptide $(10 \mathrm{~nm}$, arrows) after 90 min uptake (G, Golgi complex). Panel f depicts a second representative region rich in fusion peptide containing late endosomes/lysosomes. 
controls in the presence of three pharmacological substances to interfere with individual endocytic pathways. Chlorpromazine was used for the inhibition of clathrin-mediated internalization, MbCD for disruption of import through caveolae/lipid rafts and 5-(N-ethyl-N-isopropyl)amiloride (EIPA) for inhibition of macropinocytosis $(10,32)$. Figure S4 shows reduced viability of DCs with increasing doses of different inhibitors for determination of optimal concentrations. Of note, and as expected for these vulnerable primary cells, immature DCs without inhibitors displayed a mean viability rate of $74.2 \%$. Internalization of Alexa488-MelanA-TAT by DCs was widely blocked by $50 \mu \mathrm{g} / \mathrm{ml} \mathrm{CPZ}(P<0.001)$, whereas lower CPZ concentrations, as well as MbCD and EIPA, did not significantly reduce uptake compared with untreated DCs (Fig. 4a). Very low amounts of inhibitors showed non-significant paradoxical effects with a trend for an enhanced uptake of peptide. This observation is in accordance with earlier reports, but so far lacks a definite explanation (10,33). After establishing the optimal doses of inhibitors, internalization experiments with DCs and the Melan-A peptides or ovalbumin were performed. Alexa488Melan-A-TAT as well as Alexa488-Melan-A-polyLys uptake was downregulated by $\mathrm{CPZ}$ treatment significantly (Fig. $4 \mathrm{~b}$ ). Of note, the relative uptake was blocked to an identical extent for Alexa488-Melan-A-TAT and Alexa488-Melan-A-polyLys by all phagocytosis inhibitors (always compared with DC uptake without any inhibitors). At the same time, the absolute uptake of Alexa488-Melan-A-TAT remained twofold higher than the uptake of Alexa488-Melan-A-polyLys, as already shown for experiments without addition of inhibitors (Fig. 2). The uptake of ovalbumin by DCs was almost completely abrogated by CPZ. Interestingly, while MbCD and EIPA treatment did not reduce uptake of peptide by DCs, ovalbumin internalization was significantly decreased by MbCD. Combination of two or three inhibitors showed no significant additional effects (data not shown). In summary, Melan-A fusion peptides and ovalbumin are mainly internalized by clathrin-mediated endocytosis. Caveolae/lipid rafts-mediated endocytosis may be relevant in ovalbumin uptake in DCs to a lesser extent.

To assess whether these results also hold true for non-phagocytic cells, we studied Alexa488-Melan-A-TAT internalization by HUVECs. Similar to DCs, CPZ reduced peptide uptake whereas $\mathrm{MbCD}$ and EIPA treatment had no inhibitory effect (Fig. 4c). Still, $\mathrm{CPZ}$ alone was less effective in blocking uptake in HUVECs than in DCs $(60.9 \%$ vs $21.9 \%$ remaining uptake), and additional inhibition with EIPA significantly reduced Alexa488-Melan-A-TAT internalization in HUVECs to $28.3 \%$. Therefore, we conclude that Alexa488-Melan-A-TAT uptake by HUVECs involves other phagocytic pathways than peptide internalization by DCs, such as macropinocytosis.

\section{Discussion}

A deeper understanding of the interactions and processes that enhance and limit uptake of CPP-coupled antigens by human immature DCs seems crucial for further development of CPPs as adaptable and efficient delivery vectors. We present data in favour of a mainly energy-dependent internalization mechanism of Melan-A-TAT fusion peptides by human immature monocytederived DCs. Our results of temperature- and ATP-dependent fusion peptide uptake by DCs are in line with earlier experiments, investigating uncoupled TAT peptide uptake by HUVECs and macrophages (18). Thus, we were unable to demonstrate a significant (a)
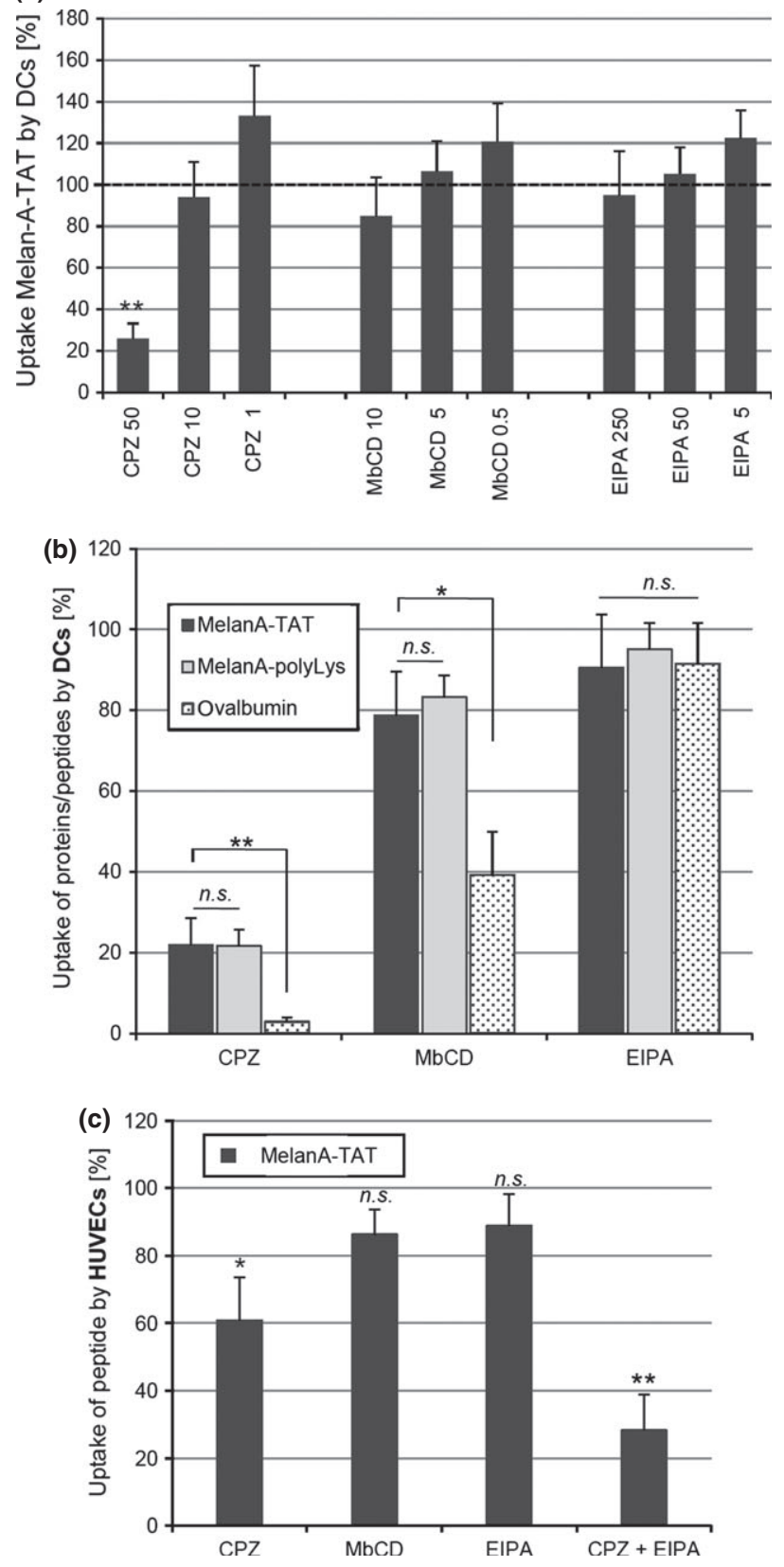

Figure 4. (a) Melan-A-TAT peptide uptake by dendritic cells (DCs) is inhibited in a concentration-dependent manner by the tested pharmacological substances; propidium iodide positive DCs have been excluded by gating procedures from this analysis ( $n=5$ independent experiments, significance level $* * P<0.01$ ). (b)

Internalization of Alexa488-Melan-A-TAT, Alexa488-Melan-A-polyLys and OVA-FITC by immature DCs in the presence of inhibitors. The MFI of internalized peptid/protein by DCs without inhibitors was set to $100 \%$ for every experiment. Concentration of inhibitors used: $50 \mu \mathrm{g} / \mathrm{ml}$ chlorpromazine, $5 \mathrm{~mm}$ methyl- $\beta$-cyclodextrin and $250 \mu \mathrm{m}$ EIPA ( $n=4$ independent experiments, significance level by unpaired $t$-test: $* P<0.05, * * P<0.01)$. (c) Internalization of Alexa488-Melan-A-TAT in the presence of inhibitors by HUVECs. Identical display of results compared with untreated controls as in $\mathrm{b}$. Values that are significantly different from controls cultured without inhibitors are marked with an asterix (paired $t$-test, $* P<0.05, * * P<0.01$ ).

role for the widely discussed energy-independent, direct penetration of the plasma membrane by inverted micelle formation, pore formation, carpet-like model or membrane thinning model $(16,23,34)$ in 
immature DCs, possibly because of the constant sampling and strong endocytotic activity of these cells. As ATP depletion inhibited the uptake of Alexa488-Melan-A-TAT only partly, a second nonenergy-dependent uptake mechanism cannot be fully excluded. However, in our colocalization studies of the internalized peptides, we did not find any conclusive evidence for an uptake mechanism outside of the endocytotic apparatus.

On encounter of any targets, myeloid phagocytes including macrophages, DCs, and neutrophils follow a serial and parallel process of 'tasting, feeling, swallowing and digesting' $(35,36)$. Most CPPs are positively charged, identifying arginine- and lysine-rich peptides as ideal vectors (10). Here, we observed a significantly higher uptake of TAT-coupled Melan-A peptide compared with polyLys-coupled Melan-A peptide by DCs despite the same net charge of these peptides. This may be explained by differences in each peptide's ability to stimulate and/or enhance uptake once bound to the surface of the phagocyte. The binding of arginine-rich peptides to sulphated sugars on the cell surface is stabilized by hydrophobic interactions and results in clustering, whereas lysine-rich peptides only interact through electrostatic attraction (19,37). In addition, our studies with HUVECs and Jurkat cells also demonstrated uptake of the fusion peptides by these non-phagocytic cells displaying the classical characteristics of CPPs, but internalization occurred to a significantly lesser extent than by DCs. As expected, ovalbumin was not internalized by HUVECs or Jurkat cells because this protein possesses no CPP characteristics.

Regarding our colocalization studies, we could demonstrate that endocytosis is a major route of Melan-A-TAT peptide internalization. It is generally accepted that the final step of phagocytosis comprises fusion of the matured phagosome with lysosomes and subsequent digestion of the target by hydrolytic enzymes in the low $\mathrm{pH}$ of the phagolysosome (35). We demonstrated localization of the fusion peptide at the earliest $90 \mathrm{~min}$ after uptake in lysosomal compartments (colocalizing with Lysotracker by fluorescence microscopy and LAMP1 by immunoelectron microscopy). These observations are also in line with previous reports suggesting a sustained cellular uptake of peptide-loaded CPPs (TAT sequence) that peaked at approximately $1-3 \mathrm{~h}$ (38). Unexpectedly, no fusion peptides were localized outside endocytic compartments of the DCs by immunoelectron microscopy. Therefore, significantly different internalization routes of TAT-coupled versus noncoupled tumor peptides into human DCs cannot conclusively explain the superior immunostimulatory effects observed by us (13) and others (reviewed in 9).

With regard to specific endocytotic routes, our findings identified clathrin-mediated endocytosis for Melan-A-TAT peptides as a major internalization mechanism by DCs. This is mainly supported by significant abrogation of Melan-A-TAT peptide uptake after treatment of DCs with CPZ, an inhibitor of clathrin-mediated endocytosis. Although specificity of the inhibitors has been an issue of debate (39), Duchardt and colleagues recently confirmed specific blockade of the different routes by extensive tracer studies (10). Of note, we found that internalization of Melan-ATAT peptide by HUVECs also involves other uptake routes because macropinocytosis seems to be involved as well, as demonstrated by significant additional EIPA inhibition of peptide uptake together with CPZ treatment of the cells.
An effective immunotherapy needs to induce strong antigenspecific $\mathrm{CD}^{+}$and $\mathrm{CD}^{+}$T-cell responses by antigen presentation on MHC class I and II molecules on the surface of antigen-presenting cells, especially DCs (40). Our results indicate that internalization of Melan-A-TAT fusion peptides is largely facilitated by clathrin-mediated endocytosis in human immature DCs. As direct penetration of peptides into cytosolic compartments of DCs would mainly result in MHC class I presentation, incorporated antigen by endocytosis ensures antigen display on MHC class II molecules as well as on MHC class I molecules by cross-presentation (41). Therefore, it can be speculated that the immune-stimulating effects from murine trials with $\mathrm{CPP}$-coupled antigens from Leishmania major or Melanoma, resulting in $\mathrm{CD}^{+} / \mathrm{CD}^{+} \mathrm{T}$ cell activation, may be transferable to humans $(26,29)$.

Considering further optimization potential of antigen delivery to immature DCs, it has been observed that the size of the particle bound to the cell surface may influence quality and quantity of the immune response, as well as accompanying mechanical stimuli $(35,42)$. As we observed significant aggregation of the Melan-ATAT fusion peptides before administration to the cells, further studies need to clarify the effect of aggregated peptide complexes on DC immunogenicity. Larger particles are more efficiently phagocytized and presented than otherwise identical smaller particles which are mainly taken up by endocytosis, although cytokine production and cellular activation level may differ $(43,44)$. Other currently investigated target properties that may influence the immune-stimulating capacities of DCs comprise the geometry, the rigidity and the topography of the target (35).

As we have evaluated the positive immune-stimulatory effects of cationic Melan-A-TAT fusion peptides in complexes with the anionic toll-like receptor three agonist Poly(i/c) for DC maturation earlier, we could demonstrate that these complexes provide a powerful tool for the generation of fully mature DCs capable of inducing a strong expansion/activation of antigen-specific $\mathrm{T}$ cells (13). Although fusion peptide aggregation was not determined at that time, we now know that this issue may have influenced earlier results. Therefore, prevention and even enhancement of peptide aggregation should be evaluated for effects on DC immunogenicity in the future. Following up on this single-step application for optimized antigen loading and maturation of DCs, further research should also include evaluation of immune-stimulatory effects of differently sized peptides with repetitive motifs of Melan-A and TAT, bound in complexes with $\operatorname{Poly}(\mathrm{i} / \mathrm{c})$.

Furthermore, intra-dermal injections of CPP-coupled immunogenic antigens without adjuvant into mice have resulted in systemic induction of a Th1-dominant immune response, as well as induction of inflammatory and CTL-mediated immune responses at the injection site (45). Looking further afield, direct injection of Melan-A-TAT fusion peptides in complexes with Poly $(\mathrm{i} / \mathrm{c})$ without the time and material consuming step of ex vivo DC generation, loading, maturation and re-injection may be worth considering in the future.

\section{Acknowledgements}

We thank A. Knüppel for excellent technical assistance and the Department of Transfusion Medicine of the University Medical Center Göttingen for supply with the leukaphereses. Furthermore, we highly appreciate the contribution of all leukapharesis donors. 
Buhl et al.

\section{Author contributions}

$\mathrm{TB}$ and $\mathrm{HAH}$ designed the research and drafted the manuscript; TB performed most experiments; SF provided HUVECs; WM performed immunoelectron microscopy; $\mathrm{LvW}$ and $\mathrm{OJ}$ designed and synthesized the peptides; NR-G and MZ conducted DLS experiments; AB, MM and MPS contributed significant intellectual input into experimental design and the manuscript.

\section{Funding}

This work was supported by a faculty grant of the University Medical Center Göttingen for young researchers to TB.

\section{Conflict of interest}

The authors have declared no conflicting interests.

\section{References}

1 Steinman R M, Banchereau J. Nature 2007: 449 419-426.

2 Finn O J. N Engl J Med 2008: 358: 2704-2715.

3 Bhargava A, Mishra D, Banerjee $S$ et al. Immunotherapy 2012: 4: 703-718.

4 Chromik J, Schnurer E, Georg Meyer R et al. J Immunol Methods 2006: 308: 77-89.

5 Joffre O P Segura E, Savina A et al. Nat Rev 2012: 12: 557-569.

6 Gromme M, Uytdehaag F G, Janssen $\mathrm{H}$ et al. Proc Natl Acad Sci USA 1999: 96: 10326 10331.

7 Verdijk P, Aarntzen E H, Lesterhuis W J et al. Clin Cancer Res 2009: 15: 2531-2540.

8 Ludewig B, McCoy K, Pericin M et al. J Immunol 2001: 166: 3678-3687.

9 Wang H Y, Wang R F. Adv Immunol 2012: 114 151-176.

10 Duchardt F, Fotin-Mleczek M, Schwarz $\mathrm{H}$ et al. Traffic 2007: 8: 848-866.

11 Dietz G P, Bahr M. Methods Mol Biol 2007 399: 181-198.

12 Shibagaki N, Okamoto T, Mitsui $\mathrm{H}$ et al. J Dermatol Sci 2011: 61: 153-161.

13 Haenssle $H$ A, Riedl P, Buhl T et al. Exp Dermatol 2010: 19: 19-28.

14 Trabulo S, Cardoso A L, Cardoso A M et al. Curr Pharm Des 2013: 19: 2895-2923.

15 Raagel H, Saalik P, Pooga M. Biochim Biophys Acta 2010: 1798: 2240-2248.

16 Madani F, Lindberg S, Langel U et al. J Biophys 2011: 2011: 414729.

17 Poon G M, Gariepy J. Biochem Soc Trans 2007 35: 788-793

18 Richard J P, Melikov K, Brooks $\mathrm{H}$ et al. J Biol Chem 2005: 280: 15300-15306.

19 Amand $\mathrm{H}$ L, Rydberg $\mathrm{H}$ A, Fornander $\mathrm{L} H$ et al. Biochim Biophys Acta 2012: 1818: 2669-2678.
20 Kaplan I M, Wadia J S, Dowdy S F. J Contro Release 2005: 102: 247-253.

21 Amand $\mathrm{H} \mathrm{L}$, Fant $\mathrm{K}$, Norden $\mathrm{B}$ et al. Biochem Biophys Res Commun 2008: 371: 621-625

22 Wadia J S, Stan R V, Dowdy S F. Nat Med 2004 10: 310-315.

23 Tunnemann G, Martin R M, Haupt $S$ et al. FASEB J 2006: 20: 1775-1784.

24 Edenhofer F. Curr Pharm Des 2008: 14: 3628 3636.

25 Foerg C, Merkle H P. J Pharm Sci 2008: 97 144-162.

26 Kronenberg K, Brosch S, Butsch F et al. J Invest Dermatol 2010: 130: 2602-2610.

27 Monini P, Cafaro A, Srivastava I K et al. PLoS ONE 2012: 7: e48781.

28 Fayolle C, Davi M, Dong H et al. Vaccine 2010: 28: 6930-6941

29 Shibagaki N, Udey M C. Eur J Immunol 2003 : 33: 850-860.

30 Buhl $\mathrm{T}$, Legler $\mathrm{T}$ J, Rosenberger $\mathrm{A}$ et al. Cancer Immunol Immunother 2012: 61: 2021-2031.

31 Haenssle H, Buhl T, Knudsen S et al. Exp Dermatol 2008: 17: 177-187.

32 Koivusalo M, Welch C, Hayashi $\mathrm{H}$ et al. J Cell Biol 2010: 188: 547-563.

33 Hawtrey A, Joubert D, van Jaarsveld $P$ et al. Drug Deliv 2002: 9: 47-53.

34 Ziegler $\mathrm{A}$, Nervi $\mathrm{P}$, Durrenberger $\mathrm{M}$ et al. Biochemistry 2005: 44: 138-148.

35 Underhill D M, Goodridge H S. Nat Rev 2012 12: 492-502.

36 Geissmann F, Gordon S, Hume D A et al. Nat Rev 2010: 10: 453-460.

37 Mitchell D J, Kim D T, Steinman L et al. J Pept Res 2000: 56: 318-325.

38 Jones $\mathrm{S}$ W, Christison $\mathrm{R}$, Bundell $\mathrm{K}$ et al. $\mathrm{Br} \mathrm{J}$ Pharmacol 2005: 145: 1093-1102.
39 Fretz $\mathrm{M}$, Jin J, Conibere $\mathrm{R}$ et al. J Control Release 2006: 116: 247-254.

40 Neefjes J, Jongsma M L, Paul P et al. Nat Rev 2011: 11: 823-836.

41 Rock K L, Shen L. Immunol Rev 2005: 207 : 166-183.

42 Ruutu M P, Chen X, Joshi O et al. Exp Dermatol 2011: 20: 534-536.

43 Brewer J M, Pollock K G, Tetley L et al. J Immunol 2004: 173: 6143-6150.

44 Rettig L, Haen S P, Bittermann A G et al. Blood 2010: 115: 4533-4541.

45 Mitsui $\mathrm{H}$, Okamoto $\mathrm{T}$, Kanzaki $\mathrm{M}$ et al. $\mathrm{Br}$ J Dermatol 2010: 162: 29-41.

\section{Supporting Information}

Additional Supporting Information may be found in the online version of this article:

Figure S1. The size (hydrodynamic radius) distribution of Alexa488-Melan-A-TAT, as revealed by DLS experiments and presented in the relative contribution to total scattering intensity.

Figure S2. Detailed FACS plots of ovalbumin-, Melan-A-polyLys- and Melan-A-TAT uptake as summarized in Fig. 2.

Figure S3. (A) Fluorescence microscopy is able to trace Alexa488-Melan-A-TAT (green) into Lysotracker (orange)-positive compartments after $90 \mathrm{~min}$ of incubation (magnification 200x). (B) Assessment of colocalization studies of earlier phagocytosis markers by fluorescence microscopy (exemplified: early endosome antigen 1 (orange), Melan-A-TAT peptide (green): upper panel after $30 \mathrm{~min}$, lower panel after $90 \mathrm{~min}$ peptide coculture)

Figure S4. Viability of DCs after treatment with inhibitors for $30 \mathrm{~min}$ was assessed by propidium iodide staining and analysis by flow cytometry. 